Wioletta Zielińska1,2 ${ }^{10}$, Agnieszka Nawrocka² ${ }^{\circledR}$, Maciej Rydzkowski², Anna Kokocha², Karolina Matulewicz ${ }^{2}$, Marta Hałas-Wiśniewska ${ }^{1,2}$, Magdalena Izdebska ${ }^{1,2}$ (D)

${ }^{1}$ Department of Histology and Embryology, Faculty of Medicine, Collegium Medicum in Bydgoszcz Nicolaus Copernicus University in Torun, Bydgoszcz, Poland

${ }^{2}$ Students research group of Cell Biology and Ultrastructure at the Department of Histology and Embryology, Nicolaus Copernicus University in Torun, Collegium Medicum in Bydgoszcz, Poland

\title{
Possibilities in the application of solid lipid nanoparticles in combination with 5-fluorouracil to overcome the drug- resistance of non-small cell lung cancer cell line A549
}

\author{
Corresponding author: \\ Wioletta Zielińska, Department \\ of Histology and Embryology, Faculty \\ of Medicine, Collegium Medicum \\ in Bydgoszcz Nicolaus Copernicus \\ University in Torun \\ Bydgoszcz, Poland \\ e-mail:wzielinska94@hotmail.com
}

Medical Research Journal 2020;

Volume 4, Number 4, 1-8

10.5603/MRJ.a2019.0037

Copyright (C) 2020 Via Medica

ISSN 2451-2591

\begin{abstract}
Introduction: Multidrug resistance of non-small cell lung cancer cells is associated with a high percentage of therapeutic failures. The aim of this study was to assess the ability of solid lipid nanoparticles as a transporter of the conventionally used cytostatic (5-fluorouracil) to overcome the resistance of A549 cells. Material and methods: MTT assay was used to assess the differences in viability of cells treated with 5-fluorouracil alone or in combination with different types of solid lipid nanoparticles. Type of cell death and distribution of cell cycle phases were evaluated using flow cytometry.

Results: The use of nanoparticles as a 5-fluorouracil transporter reduced the viability of A549 cells to a greater extent than the cytostatic alone. This was mainly due to the increase in apoptosis, but also necrosis and cell cycle arrest.

Conclusion: Our results indicate the great potential of nanotechnology in the treatment of non-small cell lung cancer. By using nanoparticles, it is possible to sensitise tumour cells to cytostatics to which they are normally resistant. In addition, literature data confirm the safety of solid lipid nanoparticle application. Key words: A549, solid lipid nanoparticles, 5-fluorouracil, drug-resistance, nanotechnology
\end{abstract}

Med Res J 2020; 5 (1): 1-8

\section{Introduction}

In spite of many available treatment methods, nonsmall cell lung cancer (NSCLC) remains a serious problem of world health care. It accounts for about $85 \%$ of all lung cancer cases, and in terms of incidence it is the first among men and the second among women. This is mainly caused by factors such as difficulties in early diagnosis, its high invasiveness, but also resistance to most of the currently available chemotherapeutic agents [1]. It drives the search for new, more effective methods of therapy for this type of cancer. One promising approach is to overcome NSCLC resistance by administering commonly used cytostatics in combination with nanoparticles.

Although nanotechnology is a relatively new branch of science, its potential application in the field of bio- technology are remarkable. Intensive research on their use in the context of sensing even small amounts of substances in analytics, diagnostics of diseases, or transport of substances into the cells is underway. It was noticed that nanoparticles had sizes similar to many of the body's biomolecules, such as receptors, antibodies, or proteins. Due to their properties, they can easily penetrate the cells but can also be modified with other molecules [2]. Nanotechnology gives the possibility to link nanoparticles and nanomaterials with currently used cytostatics, which may be helpful in more efficient transport of drugs to cells, but also constitute the basis of therapy targeted only on cancer cells [3]. In recent years, cancer nanotechnology has developed significantly. Different types of nanoparticles have been combined with conventionally used drugs 
such as doxorubicin [4], paclitaxel [5], or bleomycin [6] in in vitro studies, resulting in better response than administration of the drugs alone. Moreover, the safety and efficacy of their use was also confirmed in in vivo studies [5].

One type of nanoparticles was created for the first time in 1990: Solid Lipid Nanoparticles (SLN). They reveal advantages such as high biocompatibility and low toxicity. Also, in the case of SLN, safety of their use has been demonstrated in both in vitro and in vivo studies $[7,8]$. Moreover, they can be used via various routes of administration such as parenteral, oral, pulmonary, or rectal. However, their relatively low drug loading capacity remains a significant problem [9]. For these reasons, studies on SLN modifications with the aim of enhaning their properties are becoming more and more popular. One of the tested solutions is the use of SLN modified with a natural polysaccharide - chitosan [9]. This naturally occurring polymer also does not exhibit toxicity while regulating the release and increasing the biocompatibility of hydrophobic molecules.

One of the widely used cytostatics is 5-fluorouracil (5-FU). The compound is commonly used for head, neck, gastrointestinal, and breast cancers. Because of the resistance of NSCLC in this cancer type it is mainly applied in combined therapies, for example with cisplatin [10]. Furthermore, some studies indicate that its application often causes wide range of side effects [11]. The use of 5-FU in combination with nanoparticles potentially reduces both the resistance of the cancer cell lines and also limits the intensity of side effects by lowering the dose of the drug necessary to induce the effect.

The aim of our study was to elucidate whether the combination of 5-FU together with different SLN variants affects the resistance of the A549 non-small cell lung carcinoma cell line.

\section{Material and methods}

\section{Cell culture}

Non-small cell cancer cell line A549 was kindly provided by P. Kopiński, Ph.D. (Department of Gene Therapy, Ludwik Rydygier Collegium Medicum in Bydgoszcz, Nicolaus Copernicus University in Toruń, Poland) and cultured under standard conditions $\left(37^{\circ} \mathrm{C}, 5 \% \mathrm{CO}_{2}\right.$, humidified atmosphere) in Dulbecco's modified Eagle's medium (DMEM; Sigma-Aldrich, St., Louis, MO, USA) with the addition of $10 \%$ foetal bovine serum (FBS; Sigma-Aldrich, St., Louis, MO, USA) and antibiotics $(50 \mu \mathrm{g} / \mathrm{ml}$ of gentamycin; Sigma-Aldrich, St., Louis, MO, USA). The cells were grown as a monolayer in 6- or 12-well plates or culture flasks, depending on the experiment. After reaching $70-80 \%$ confluence the cells were treated for $24 \mathrm{~h}$ with nanoparticles containing 5-fluorouracil (5-FU; Sigma-Aldrich, St., Louis, MO, USA) to obtain $0.5,1,1.5,2$, and $4 \mathrm{mM}$ concentrations of the drug. Additionally, two types of control cells were applied. The first were cells cultured in clean culture medium, while the second comprised cells treated with empty nanoparticles (without 5-FU addition) at the same concentration as for the study group. During the time of experiment regular tests for mycoplasma infections based on rapid uptake of the DAPI (Sigma-Aldrich) by cellular DNA were conducted and found to be negative [12].

\section{Nanoparticles preparation}

The nanoparticles were prepared on the basis of the modified protocol presented by Xiao-Ying et al. [9]. In brief, monostearin (MON; Sigma-Aldrich, St., Louis, $\mathrm{MO}$, USA) was dissolved in DMSO at $75^{\circ} \mathrm{C}$, while chitosan oligosaccharide (CSO; Sigma-Aldrich) was dissolved in water at room temperature (RT). The monostearin was then mixed with 5-FU in DMSO and heated again. The resulting solution was suspended in water (MON, SLN), water with chitosan ( $\mathrm{CH}$, SLN modified with chitosan), or water with chitosan oligosaccharide and various amounts of glutaraldehyde ( $1 \mu \mathrm{M}$ GA or $3 \mu \mathrm{M}$ GA, cross-linked SLN modified with chitosan oligosaccharide). Next, the nanoparticle solutions were mixed intensively for six hours and frozen overnight after acidification with $1 \mathrm{M} \mathrm{HCl}$. The next day, the nanoparticle solutions were thawed and centrifuged (15,000 rpm, $45 \mathrm{~min}$.). The resulting precipitate was washed with phosphate-buffered saline (PBS), centrifuged again, and resuspended in PBS. The control nanoparticles were prepared according to the same protocol but with the addition of DMSO instead of 5-FU solution.

The assessment of encapsulation efficiency and drug loading capacity

In the first stage, a standard curve for 5-FU at a wavelength of $267 \mathrm{~nm}$ was obtained by measuring the absorbance of increasing concentrations of cytostatic. From the prepared samples $100 \mu \mathrm{l}$ was taken and added to $900 \mu \mathrm{l}$ of DMSO. The samples were heated for $45 \mathrm{~min}$ at $37^{\circ} \mathrm{C}$ and centrifuged. The absorbance of the supernatant was measured by a spectrophotometer (Spectra Academy, K-MAC, Korea). The 5-FU content in the supernatant was determined from the standard curve. Drug loading (DL) and encapsulation efficiency (EE) were calculated based on the following formulas:

$$
\begin{gathered}
E E=\left(\frac{W_{5-F U}-W_{S}}{W_{5-F U}}\right) \times 100 \% \\
D L=\left(\frac{W_{5-F U}-W_{S}}{W_{5-F U}-W_{S+} W_{M+} W_{C S O}}\right) \times 100 \%
\end{gathered}
$$

$\mathrm{W}_{5-\mathrm{FU}}$ - theoretical 5-FU concentration

$\mathrm{W}_{\mathrm{S}}$ - measured 5-FU concentration in the supernatant

$\mathrm{W}_{\mathrm{M}}$ - concentration of monostearin 
$\mathrm{W}_{\mathrm{cso}}-$ concentration of chitosan

MTT assay

The cell viability was assessed using MTT assay. For this purpose, A549 was grown in 12-well plates and treated with selected concentrations of 5-FU loaded SLN or 5-FU alone $(0.5,1,1.5,2,4 \mathrm{mM})$ for $24 \mathrm{~h}$. Then, the $5 \mathrm{mg} / \mathrm{ml}$ MTT solution (Sigma-Aldrich) was prepared and mixed with DMEM without phenol red (Lonza, Basel, Switzerland) at the ratio of 1:9. Next, the solution was added to the cells for $3 \mathrm{~h}$. After this time, the resultant purple formazan crystals were dissolved in isopropanol (10 $\mathrm{min}, 37^{\circ} \mathrm{C}$; Avantor, Gliwice, Poland) and centrifuged at 13,000 RPM for $2 \mathrm{~min}$. The intensity of the violet colour of the samples corresponding to the cell viability was measured with a spectrophotometer (Spectra Academy) at $570 \mathrm{~nm}$ wavelength. The results obtained for appropriate controls were assumed as $100 \%$. Moreover, on the basis of obtained data the half maximal inhibitory concentration $\left(\mathrm{IC}_{50}\right)$ was estimated using CompuSyn software [13].

\section{Cell death analysis}

In order to evaluate the mechanism of cell death underlying the reduction of cell viability, double staining with annexin $\mathrm{V}$ and propidium iodide (AV, PI; Invitrogen, Life Technologies, Thermo Fisher Scientific, Inc., Waltham, MA, USA) was used. The obtained results allowed the distinction of live (AV-/PI-), early apoptotic $(\mathrm{AV}+/ \mathrm{PI}-)$, late apoptotic $(\mathrm{AV}+/ \mathrm{PI}+)$, and necrotic $(\mathrm{AV}-\mathrm{PI}+)$ cell populations. The whole procedure was performed according to the manufacturer's protocol. Briefly, the A549 cells were cultured on six-well plates with the addition of 5-FU loaded SLN or 5-FU alone for $24 \mathrm{~h}$. Next, the cells were trypsinised, centrifuged, resuspended in $100 \mu$ l of Annexin Binding Buffer (ABB; Invitrogen/Life Technologies), and then $5 \mu$ l of Annexin $\checkmark$ Alexa Fluor 488 was added for $20 \mathrm{~min}$. Then, cells were centrifuged again, resuspended in $100 \mu \mathrm{l}$ of ABB, and incubated with $1 \mu \mathrm{l}$ of PI for $3 \mathrm{~min}$. The obtained data were analysed using a Guava easyCyte $6 \mathrm{HT}-2 \mathrm{~L}$ Benchtop Flow Cytometer (Merck Milipore, Burlington, MA, USA) and FlowJo vX0.7 software (FlowJo LLC, Ashand, OR, USA).

\section{Cell cycle assessment}

The distribution of the cell cycle phases of cells treated with 5-FU loaded SLN and 5-FU alone was evaluated using a flow cytometer and Tali Cell Cycle Kit (Invitrogen/Life Technologies, Thermo Fisher Scientific, Inc., Waltham, MA, USA) according to the manufacturer's instructions. After $24 \mathrm{~h}$ of treatment the cells were trypsinised, centrifuged, fixed with ice-cold $70 \%$ ethanol, and refrigerated overnight. The next day, the cells were centrifuged, washed with PBS, centrifuged again, resuspended in the Tali Cell Cycle Solution, and incubated for $30 \mathrm{~min}$. The results were analysed using a Guava easyCyte 6HT-2L Benchtop Flow Cytometer (Merck Milipore). The percentage of cells in each cell cycle phase was assessed by FlowJo vX0.7 software (FlowJo LLC).

\section{Statistical analysis}

The results were considered significant when $P<0.05$. The statistical analysis was carried out using GraphPad Prism 6 (GraphPad Software, Inc., La Jolla, CA, USA). The MTT assay results were analysed with the nonparametric Wilcoxon test assuming the control as $100 \%$. The cell death was assessed using Kruskal-Wallis test, and the cell cycle was evaluated by two-way ANOVA test. Statistically significant differences are marked with an asterisk.

\section{Results}

\section{Encapsulation efficiency and drug-loading capacity}

In order to assess encapsulation efficiency and drug-loading capacity spectrophotometric measurements at a wavelength of $267 \mathrm{~nm}$ were conducted and then aligned to a standard curve. The obtained values were similar for all SLN modifications used. The mean value calculated from five independent measurements of encapsulation efficiency was $95.83 \%$ for unmodified SLN (MON), 91.77\% for chitosan-modified nanoparticles (CSO), 94.79\% for chitosan-modified nanoparticles with $1 \mu \mathrm{M}$ glutaraldehyde ( $1 \mathrm{GA}$ ), and $94.81 \%$ for those with the addition of $3 \mu \mathrm{M}$ glutaraldehyde ( $3 \mathrm{GA}$ ). In turn, the drug-loading capacity, determined in a similar way, reached $75.83 \%$ (MON), 71.82\% (CSO), 74.82\% (1 GA), and $74.83 \%$ ( $3 \mathrm{GA})$, respectively. All of the mentioned results are shown in Figure 1.

\section{MTT assay}

In order to evaluate the effect of 5-FU alone and the cytostatic encapsulated in the nanoparticles MTT assay was performed. The results are shown in Figure 2. In all cases, a dose-dependent decrease in the number of viable cells was observed. For 5-FU after $24-\mathrm{h}$ incubation with cytostatic, values of $80.37 \%$, $69.54 \%, 67.26 \%, 54.17 \%$, and $44.94 \%$ were obtained for subsequent doses of $0.5,1,1.5,2$, and $4 \mu \mathrm{M} 5$-FU, respectively (Fig. 2A). In the case of unmodified SLN, the number of living cells was significantly reduced only in the highest dose in comparison to 5-FU alone, and the 


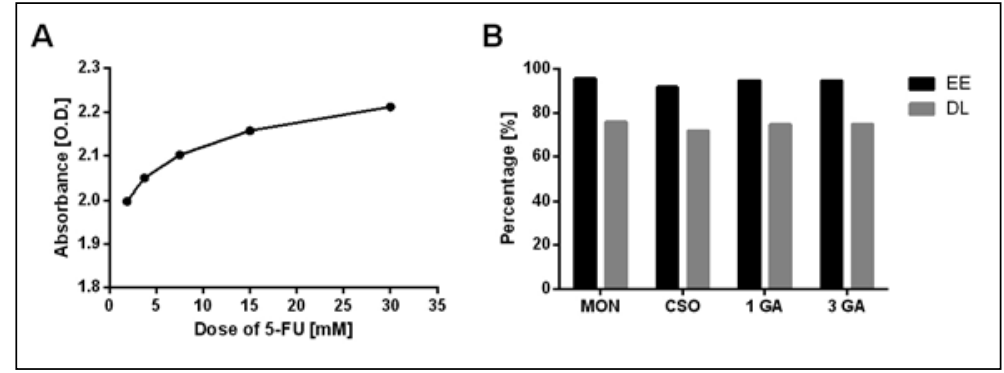

Figure 1. Encapsulation efficiency and drug-loading capacity. A. A standard curve determining the dependence of absorbance on the concentration of 5-FU measured at a wavelength of $266 \mathrm{~nm}$ used to assess the EE\% (encapsulation efficiency) and DL\% (drug-loading capacity) of types of nanoparticles used. B. The encapsulation efficiency (EE\%) and drug-loading capacity (DL\%). The mean value calculated from five independent measurements of encapsulation efficiency for unmodified SLN (MON), chitosan-modified nanoparticles (CSO), chitosan-modified nanoparticles cross-linked with $1 \mu \mathrm{M}$ glutaraldehyde (1 GA), and chitosan-modified nanoparticles cross-linked with $3 \mu \mathrm{M}$ glutaraldehyde (3 GA)

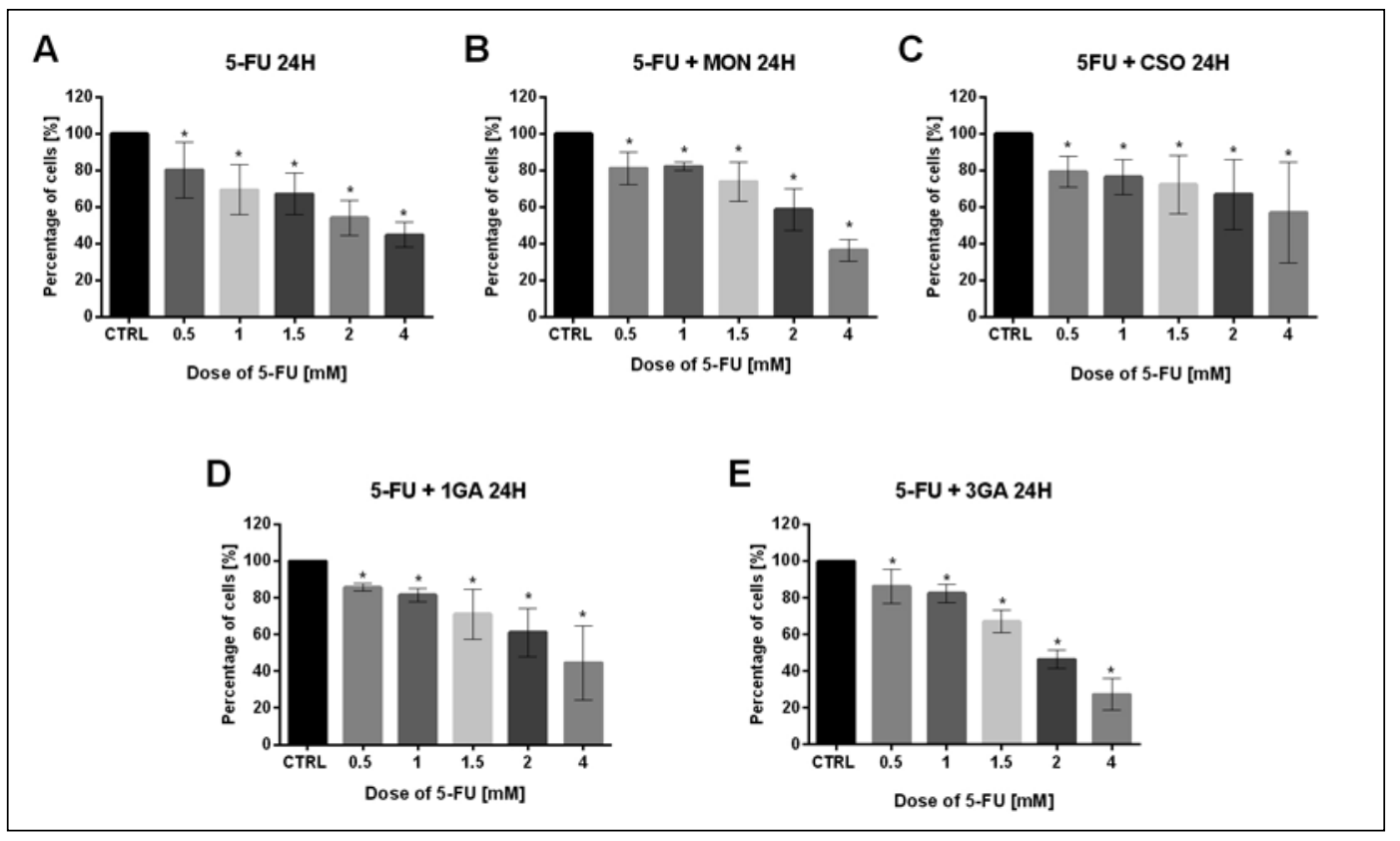

Figure 2. Analysis of A549 cell viability. The MTT assay was performed to evaluate the effect of 5-FU alone and the cytostatic encapsulated in the nanoparticles after $24 \mathrm{~h}$. A549 cells were treated with different concentrations $(0.5,1$, 1.5, 2, 4 mM) of: A) 5-FU; B) 5-FU-loaded unmodified SLN (5-FU + MON); C) 5-FU-loaded chitosan-modified SLNs (5-FU + CSO); D) 5-FU-loaded chitosan-modified SLNs crosslinked with $1 \mu \mathrm{M}$ glutaraldehyde (5-FU + $1 \mathrm{GA})$; and E) 5-FU-loaded chitosan-modified SLNs crosslinked with $3 \mu \mathrm{M}$ glutaraldehyde (5-FU $+3 \mathrm{GA})$. The data were represented by mean \pm SD. “*” $p<0.05$ vs. untreated cells (CTRL)

percentage for all doses amounted to $81.18 \%, 82.28 \%$, $73.92 \%, 58.63 \%$, and $36.47 \%$, respectively (Fig. 2B). For chitosan-modified nanoparticles, the limiting effect on the A549 line resistance to 5-FU was not observed as the percentage of viable cells was $79.45 \%, 76.51 \%$, $72.38 \%, 67.0 \%$, and $57.28 \%$ for successively increasing doses (Fig. 2C). A similar situation was noted in the case of nanoparticles modified with chitosan with the addition of glutaraldehyde as a cross-linking agent.
At a lower concentration of glutaraldehyde, the difference between the effectiveness of nanoparticles and 5 -FU was not confirmed, and the cell viability reached 85.66\%, 81.56\%, 71.14\%, 61.16\%, and 44.71\% (Fig. 2D). In turn, the use of higher glutaraldehyde concentration at the highest doses of 5-FU (2 and $4 \mu \mathrm{M})$ resulted in a decrease in viability to a greater degree than for 5 -FU alone; the percentage of living cells was $86.31 \%$, $82.4 \%, 67,07 \%, 46.54 \%$, and $27.48 \%$ for $0.5,1,1.5,2$, 


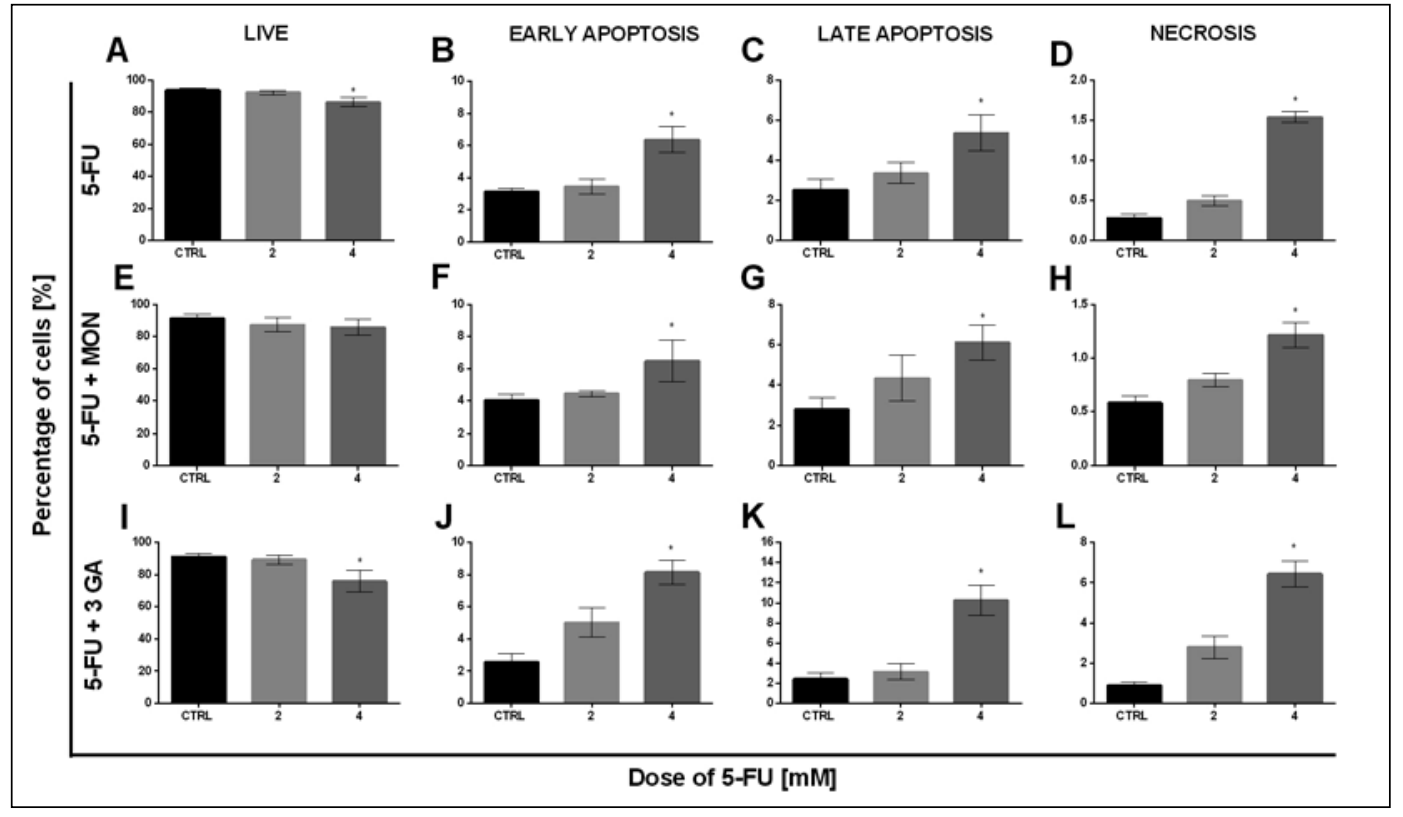

Figure 3. Analysis of cell death. The double staining of annexin V (AV) and propidium iodide (PI) was used to evaluate the type of cell death. A549 cells were treated with 2 and $4 \mathrm{mM}$ of 5-FU, 5-FU-loaded unmodified SLNs (5-FU + MON), and 5-FU loaded chitosan-modified SLNs crosslinked with $3 \mu \mathrm{M}$ glutaraldehyde (5-FU $+3 \mathrm{GA})$. A, E, I-the percentage of live cells (AV-/PI-); B, F, J - the percentage of early apoptotic cells (AV+/PI-); C, G, K-the percentage of late apoptotic cells $(A V+/ P I+) ; D, H, L-$ the percentage of necrotic cells $(A V-/ P I+)$. The data were represented by mean $\pm S D$. "*" $p<0.05$ vs. untreated cells (CTRL)

and $4 \mu \mathrm{M}$ doses of 5-FU, respectively, in comparison to control (Fig. 2E). At the same time, there was no significant effect of the nanoparticles themselves on cell survival, which confirms the safety of their use. Because the most promising results were obtained for only two SLN types - unmodified SLN and chitosan-modified SLN cross-linked with $3 \mu \mathrm{M}$ GA linked with 2 and $4 \mu \mathrm{M}$ doses of 5-FU - the study group in further experiments was limited only to them.

\section{Cell death analysis}

In order to elucidate the effect of 5-FU and the cytostatic encapsulated in the SLN, double staining with Annexin $\mathrm{V}$ and propidium iodide was performed. The results showed that for 5-FU alone the amount of live cells dropped from $93.75 \%$ in the control to $92.05 \%$ for $2 \mathrm{mM}$ and $86.11 \%$ for $4 \mathrm{mM}$ doses of the compound (Fig. $3 A)$. The decrease in the percentage of live cells was caused mainly by apoptotic cell death; $3.13 \%$ (CTRL), $3.45 \%$ (2 mM 5-FU), and 6.37\% (4 mM 5-FU) cells were identified as early-apoptotic cells, and $2.54 \%$ (CTRL), $3.36 \%$ (2 mM 5-FU), and 5.36\% (4 mM 5-FU) as late apoptotic (Fig. 3B, C). There was also a slight increase in the percentage of necrotic cells, from $0.28 \%$ (CTRL) to 0.5\% (2 mM 5-FU) and 1.54\% (4 mM 5-FU) (Fig. 3D). For unmodified SLN, the percentages were, respectively, 91.27\% (CTRL), 87.4\% (2 mM), and 85.74\% (4 mM) for live, $4.04 \%$ (CTRL), 4.44\% (2 mM), and 6.48\% (4 mM) for early apoptotic, and $2.79 \%$ (CTRL), $4.32 \%$ (2 mM), and $6.11 \%(4 \mathrm{mM}$ ) for late apoptotic cells (Fig. 3E-G). Similarly as for $5-\mathrm{FU}$ alone, the increase in the percentage of necrotic cells from $0.58 \%$ through $0.78 \%$ to $1.21 \%$ was observed with the increase in the cytostatic dose (Fig. $3 \mathrm{H})$. However, the biggest differences were observed in the case of SLN modified with chitosan and cross-linked with $3 \mu \mathrm{M}$ concentration of glutaraldehyde. Values of $91.35 \%, 89.3 \%$, and $75.9 \%$ were obtained for live cells, respectively (Fig. 3I). Again, the decrease in cell survival was mainly due to an increase in the percentage of cells, both early $(2.58 \%, 5.04 \%, 8.16 \%$, respectively) and late $(2.4 \%, 3.16 \%, 10.26 \%$, respectively) apoptotic cells (Fig. $3 \mathrm{~J}, \mathrm{~K}$ ). Additionally, enhanced necrotic cell death was observed, which resulted in an increase in the percentage of this population from $0.88 \%$ through $2.8 \%$ to $6.44 \%$ (Fig. 3L). In all three groups, only values obtained for the highest dose (4 $\mathrm{mM}$ ) were considered as statistically significant. At the same time, in the case of cells treated with empty nanoparticles (without the addition of 5-FU), no significant differences comparing to the control cells treated with empty nanoparticles were confirmed (data not shown). 


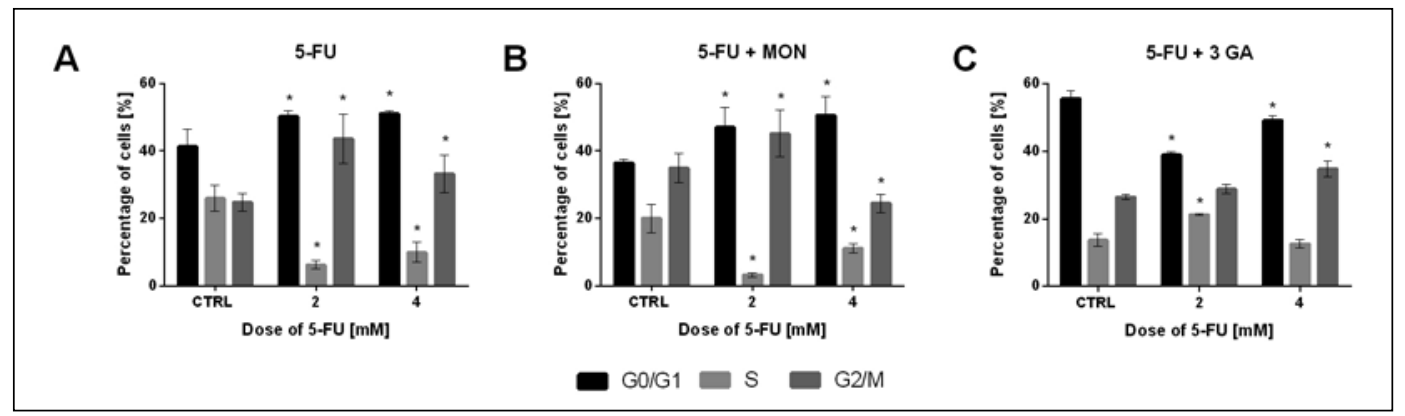

Figure 4. Analysis of cell cycle. The Tali Cell Cycle Kit was used to analyse the distribution of the cell cycle phases of A549 cells treated with 2 and 4 mM of: A) 5-FU ; B) 5-FU-loaded unmodified SLNs (5-FU + MON); and C) 5-FU-loaded chitosan-modified SLNs crosslinked with $3 \mu \mathrm{M}$ glutaraldehyde (5-FU + $3 \mathrm{GA})$. Figure presenting the percentage of A549 cells population in G0/G1, S, and G0/G2 phases. Data represented by mean \pm SD. "*” $p<0.05$ vs. untreated cells (CTRL)

\section{Cell cycle assessment}

Cell cycle phase evaluation was performed using flow cytometry. 5-FU alone caused an increase in the percentage of cells in the G0/G1 and G2/M phases comparing to untreated control with the values as follows: $41.33 \%$ (CTRL), 50.2\% (2 mM 5-FU), and $51.1 \%$ (4 mM, 5-FU) in the G0/G1 phase. In turn, for $\mathrm{G} 2 / \mathrm{M}$ the values reached $24.77 \%$ for control, $43.63 \%$ for $2 \mathrm{mM}$, and $33.23 \%$ for $4 \mathrm{mM}$. However, in the $S$ phase a decrease was observed dropping from $25.93 \%$ for control cells to $6.13 \%$ for $2 \mathrm{mM}$ and $9.95 \%$ for $4 \mathrm{mM}$ doses of the cytostatic. All of the described changes were statistically significant (Fig. 4A). For unmodified SLN loaded with 5-FU the distribution of cell cycle phases was similar as for the 5-FU alone. We observed higher percentage of cells in the G0/G1 phase, estimated as $36.4 \%$ for control, $46.97 \%$ for $2 \mathrm{mM}$, and $50.47 \%$ for $4 \mathrm{mM}$ doses of $5-F U$. In turn, for the $S$ phase the reduction in the cell number was confirmed from $19.97 \%$ for control, $3.22 \%$ for $2 \mathrm{mM}$, and $11.03 \%$ for $4 \mathrm{mM}$, in comparison to the control cells treated with empty SLN. For the G2/M phase, the effect varied between the doses. An increase from $34.93 \%$ to $45.17 \%$ was observed for the $2 \mathrm{mM}$ dose, while a decrease to $24.42 \%$ for $4 \mathrm{mM}$ was confirmed (Fig. 4B). The cell cycle phase distribution undergoes a significant change in the cells treated with SLN modified with chitosan crosslinked with 3GA. The number of cells with a DNA level corresponding to the G0/G1 phase decreased from $55.6 \%$ for control to $38.93 \%$ for $2 \mathrm{mM}$ and $49.17 \%$ for $4 \mathrm{mM}$ of the compound. For the S phase, $13.7 \%$ for control, $21.23 \%$ for $2 \mathrm{mM}$, and $12.5 \%$ for $4 \mathrm{mM}$ dose of $5-\mathrm{FU}$ was noted. In contrast, $26.43 \%, 28.8 \%$, and $34.8 \%$ were observed for cells in the G2/M phase, for control, and increasing doses, respectively (Fig. $4 \mathrm{C}$ ).

\section{Discussion}

Nanotechnology is a promising tool for the development of many branches of science. It is also applied in the field of medical science. It may be particularly useful in the context of oncology. Potentially, nanoparticles may be employed not only as a factor increasing the effectiveness of therapy but also as a base for targeted therapy. At the same time, the increase in effectiveness may be achieved in spite of lowering cytostatics doses, which will probably limit side effects. It would also be possible to treat cancers known to be resistant to certain types of cytostatics.

5-FU is a relatively well-known and widely used cytostatic. Unfortunately, some types of tumours are resistant to the drug due to the increased expression of $\mathrm{Bcl}-2, \mathrm{Bcl}-\mathrm{XL}$, and $\mathrm{Mcl}-1$ genes, hyperactivity of deoxyuridine triphosphatase, abnormal methylation of the MLH1 gene or CpG Islands, altered drug influx and efflux, and enhanced drug inactivation [14-19]. Studies have shown that nanoparticles coated with $5-\mathrm{FU}$ can limit some of these effects. The greater efficiency of 5-FU-loaded nanoparticles relative to the administration of the cytostatic alone has been proven in both in vitro [7] and in vivo [6] studies. This is also consistent with our results because the solution of empty nanoparticles did not cause any changes in cell viability or cell cycle in comparison to untreated A549 cells.

The method we propose is a combination of $5-\mathrm{FU}$ and SLNs. There are several literature reports suggesting a great potential of such a combination [19-21]. Use of SLNs has a lot of advantages because, for example, they can be loaded with both hydrophobic and hydrophilic compounds, and their preparation usually does not involve any toxic solutions [22]. Moreover, this approach is relatively easy to apply; however, the stability of the obtained nanoparticles is considered to be low [9]. This imposes the need to introduce modifications 
to enhance the durability of this type of nanoparticles. In accordance with our knowledge, this is the first case of using SLN prepared by solvent diffusion method with chitosan and glutaraldehyde as a crosslinking agent in combination with 5-FU. SLNs prepared with this method have a negative charge, which, after adding an aqueous solution of chitosan, enabled its adsorption on the surface of nanoparticles by generating electrostatic interactions. Unfortunately, the study of the efficiency of encapsulation and the cytotoxic effect on cells of the A549 line showed a decrease in comparison to unmodified SLN. This is in contrast to the commonly obtained result; usually the coating of nanoparticles with chitosan increases their stability [9]. This was probably caused by the use of chitosan lactate instead of chitosan, and the difference in the chemical properties of both molecules. The results obtained indicate that the use of chitosan lactate may interfere with 5-FU binding by nanoparticles. However, cross-linking nanoparticles modified with chitosan under the influence of glutaraldehyde increases their stability. This resulted in enhanced encapsulation efficiency and cytotoxic effect with growing GA concentration. The same observations were made by Xiao-Ying et al., who also confirmed an increase in the efficiency of encapsulation due to crosslinking of SLNs modified with chitosan using GA [9]. The results were also confirmed by cell survival analysis, which was found to be lower for SLNs cross-linked with $3 \mu \mathrm{M}$ GA than for any other nanoparticle group. At the same time, the demonstrated higher efficiency of SLNs modified with chitosan and cross-linked with $3 \mu \mathrm{M}$ GA was not associated with differences in parameters such as drug-loading capacity or encapsulation efficiency, as they were very similar for all types of nanoparticles used.

The observed decrease in cell viability was caused mainly by apoptosis and cell cycle arrest, and to a lower extent also by necrosis. All of these phenomena were intensified after treatment with drug entrapped in SLNs modified with chitosan and cross-linked with $3 \mu \mathrm{M}$ GA. The increase in the apoptotic cell death in cancer cells is one of the most desirable effects of anti-tumour compounds due to the lack of inflammation induction by this type of death [23]. Moreover, addition of SNLs changed the cell cycle phase distribution especially in the case of SLNs modified with chitosan and cross-linked with $3 \mu \mathrm{M}$ GA. Alterations in cell cycle after encapsulation of the drug in different types of nanoparticles including SLNs were also confirmed by available literature reports [24, 25]. All of this suggests that the use of modified SLNs loaded with 5-FU may be beneficial in the treatment of 5-FU-resistant non-small cell lung cancer. The next step in research should be the assessment of the selective effect of designed nanoparticles on cancer cells by comparing them to the impact on normal lung cells.

\section{Conclusion}

The obtained data suggest that SLNs modified with chitosan and cross-linked with GA may be useful in overcoming the resistance of non-small cell lung cancer to 5-FU. Additionally, nanoparticles seem to be not only an effective but also a safe tool, which requires confirmation also on normal cell lines. Furthermore, the effectiveness of SLNs may be improved by introduction of modifications in their design such as coating with additional substances or cross-linking.

\section{Conflict of interest: None declared}

Acknowledgments: This study was supported by a research task within the framework of the statutory activities and Student Researches (Nicolaus Copernicus University in Torun, Faculty of Medicine, Collegium Medicum in Bydgoszcz).

\section{References}

1. Molina JR, Yang P, Cassivi SD, et al. Non-small cell lung cancer: epidemiology, risk factors, treatment, and survivorship. Mayo Clin Proc. 2008 83(5): 584-594, doi: 10.4065/83.5.584, indexed in Pubmed: 18452692

2. Wang EC, Wang AZ. Nanoparticles and their applications in cell and molecular biology. Integr Biol (Camb). 2014; 6(1): 9-26, doi: 10.1039/c3ib40165k, indexed in Pubmed: 24104563.

3. Allegra A, Penna G, Alonci A, et al. Nanoparticles in oncology: the new theragnostic molecules. Anticancer Agents Med Chem. 2011; 11(7): 669-686, doi: 10.2174/187152011796817682, indexed in Pubmed: 21787275.

4. Zheng G, Zheng M, Yang B, et al. Improving breast cancer therapy using doxorubicin loaded solid lipid nanoparticles: Synthesis of a novel arginine-glycine-aspartic tripeptide conjugated, $\mathrm{pH}$ sensitive lipid and evaluation of the nanomedicine in vitro and in vivo. Biomed Pharmacother. 2019; 116: 109006, doi: 10.1016/j.biopha.2019.109006, indexed in Pubmed: 31152925.

5. Chowdhury P, Nagesh PKB, Hatami E, et al. Tannic acid-inspired paclitaxel nanoparticles for enhanced anticancer effects in breast cancer cells. J Colloid Interface Sci. 2019; 535: 133-148, doi: 10.1016/j. jcis.2018.09.072, indexed in Pubmed: 30292104

6. Yang C, Uertz J, Chithrani DB. Colloidal Gold-Mediated Delivery of Bleomycin for Improved Outcome in Chemotherapy. Nanomaterials (Basel). 2016; 6(3), doi: 10.3390/nano6030048, indexed in Pubmed: 28344305.

7. Clemente N, Ferrara B, Gigliotti CL, et al. Solid Lipid Nanoparticles Carrying Temozolomide for Melanoma Treatment. Preliminary In Vitro and In Vivo Studies. Int J Mol Sci. 2018; 19(2), doi: 10.3390/ijms19020255, indexed in Pubmed: 29364157.

8. Shi S, Zhou M, Li X, et al. Synergistic active targeting of dually integrin avB3/CD44-targeted nanoparticles to B16F10 tumors located at different sites of mouse bodies. J Control Release. 2016; 235: 1-13, doi: 10.1016/j.jconrel.2016.05.050, indexed in Pubmed: 27235150.

9. Ying $X Y, C u i D, Y u L$, et al. Solid lipid nanoparticles modified with chitosan oligosaccharides for the controlled release of doxorubicin. Carbohydrate Polymers. 2011; 84(4): 1357-1364, doi: 10.1016/j. carbpol.2011.01.037.

10. Kucuk O, Shevrin DH, Pandya KJ, et al. Phase II trial of cisplatin etoposide, and 5-fluorouracil in advanced non-small-cell lung cancer: an Eastern Cooperative Oncology Group Study (PB586). Am J Clin Oncol. 2000; 23(4): 371-375, doi: 10.1097/00000421-20000800000012, indexed in Pubmed: 10955866.

11. Papanastasopoulos P, Stebbing J. Molecular basis of 5-fluorouracil-related toxicity: lessons from clinical practice. Anticancer Res. 2014; 34(4): 1531-1535, indexed in Pubmed: 24692679. 
12. Jung $\mathrm{H}$, Wang SY, Yang IW, et al. Detection and treatment of mycoplasma contamination in cultured cells. Chang Gung Med J. 2003; 26(4) 250-258, indexed in Pubmed: 12846524

13. Chou TC, Martin N. CompuSyn for drug combinations: PC Software and User's Guide: a computer program for quantitation of synergism and antagonism in drug combinations, and the determination of IC50 and ED50 and LD50 values. Paramus (NJ): ComboSyn. ; 2005.

14. Wu DW, Huang CC, Chang SW, et al. BCl-2 stabilization by paxillin confers 5-fluorouracil resistance in colorectal cancer. Cell Death Differ. 2015; 22(5): 779-789, doi: 10.1038/cdd.2014.170, indexed in Pubmed: 25323586.

15. Akagi $H$, Higuchi $H$, Sumimoto $H$, et al. Suppression of myeloid cel leukemia-1 (Mcl-1) enhances chemotherapy-associated apoptosis in gastric cancer cells. Gastric Cancer. 2013; 16(1): 100-110, do: 10.1007/s10120-012-0153-6, indexed in Pubmed: 22527182.

16. Jover R, Nguyen TP, Pérez-Carbonell L, et al. 5-Fluorouracil adjuvan chemotherapy does not increase survival in patients with $\mathrm{CpG}$ island methylator phenotype colorectal cancer. Gastroenterology. 2011 140(4): 1174-1181, doi: 10.1053/j.gastro.2010.12.035, indexed in Pubmed: 21185836.

17. Gu W, Fang FF, Li B, et al. Characterization and resistance mechanisms of a 5-fluorouracil- resistant hepatocellular carcinoma cell line. Asian Pac J Cancer Prev. 2012; 13(9): 4807-4814, doi 10.7314/apjcp.2012.13.9.4807, indexed in Pubmed: 23167424

18. Kawahara A, Akagi $Y$, Hattori S, et al. Higher expression of deoxyuridine triphosphatase (dUTPase) may predict the metastasis po- tential of colorectal cancer. J Clin Pathol. 2009; 62(4): 364-369, doi: 10.1136/jcp.2008.060004, indexed in Pubmed: 19052026.

19. Zhang N, Yin Y, Xu SJ, et al. 5-Fluorouracil: mechanisms of resistance and reversal strategies. Molecules. 2008; 13(8): 1551-1569, doi: 10.3390/molecules13081551, indexed in Pubmed: 18794772

20. Du B, Yan Y, Li Y, et al. Preparation and passive target of 5-fluorouracil solid lipid nanoparticles. Pharm Dev Technol. 2010; 15(4): 346-353, doi: 10.3109/10837450903246390, indexed in Pubmed: 19769532.

21. Khallaf RA, Salem HF, Abdelbary A. 5-Fluorouracil shell-enriched solid lipid nanoparticles (SLN) for effective skin carcinoma treatment. Drug Deliv. 2016; 23(9): 3452-3460, doi: 10.1080/10717544.2016.1194498, indexed in Pubmed: 27240935

22. Thukral DK, Dumoga S, Mishra AK. Solid lipid nanoparticles: promising therapeutic nanocarriers for drug delivery. Curr Drug Deliv. 2014 11(6): 771-791, doi: 10.2174/156720181106141202122335, indexed in Pubmed: 25469779.

23. Pfeffer CM, Singh ATK. Apoptosis: A Target for Anticancer Therapy Int J Mol Sci. 2018; 19(2), doi: 10.3390/ijms19020448, indexed in Pubmed: 29393886

24. Li Q, Huang C, Liu L, et al. Effect of Surface Coating of Gold Nanoparticles on Cytotoxicity and Cell Cycle Progression. Nanomaterials (Basel). 2018; 8(12), doi: 10.3390/nano8121063, indexed in Pubmed: 30562921.

25. Yuan Q, Han J, Cong W, et al. Docetaxel-loaded solid lipid nanoparticles suppress breast cancer cells growth with reduced myelosuppression toxicity. Int J Nanomedicine. 2014; 9: 4829-4846, doi: 10.2147/IJN. S70919, indexed in Pubmed: 25378924 